\title{
Correlación entre los Factores de Riesgo Intralaboral y los Niveles Séricos de Cortisol en Docentes Universitarios
}

\section{CORRELATION BETWEEN INTRA-LABOR RISK FACTORS AND SERUM LEVELS OF CORTISOL IN PROFESSORS}

Álvaro L. Fajardo-Zapata1, Fernando Montejo-Angel' ${ }^{2}$ Germán Molano-Vargas ${ }^{3}$, Jenny Hernández-Niño ${ }^{4}$, Adelaida Quintero-Guzmán ${ }^{5}$

1. Magister en Toxicologia Universidad Nacional de Colombia. Especialista en Administración en Salud Ocupacional Universidad JTL. Médico Cirujano Universidad Nacional de Colombia. Ingeniero Industrial Universidad Distrital. Profesor asociado de la Fundación Universitaria del Área Andina sede Bogotá.

2. Magister en Psicologia Universidad Nacional de Colombia. Especialista en Gerencia de proyectos educativos Universidad Distrital. Psicólogo Universidad Nacional de Colombia. Director de unidad de la Fundación Universitaria del Área Andina sede Bogotá.

3. Magister en Educación Universidad Pedagógica. Especialista en Gerencia de la salud ocupacional Universidad del Rosario. Especialista en Epidemiología Universidad del Rosario. Médico Universidad Nacional de Colombia. Profesor de la Fundación Universitaria del Área Andina sede Bogotá.

4. Magister en Microbiología Universidad Javeriana. Especialista en Docencia Universitaria Universidad Santo Tomas. Bacterióloga Colegio mayor de Cundinamarca. Profesora de la Fundación Universitaria del Área Andina sede Bogotá.

5. Magister en Economía Universidad de los Andes. Estadista Universidad Nacional de Colombia. Profesora de la Fundación Universitaria del Área Andina sede Bogotá.

\section{RESUMEN}

Introducción: los profesores universitarios se enfrentan a diferentes factores generadores de estrés laboral. Objetivo: explorar la asociación entre los factores de riesgo psicosocial intralaboral y los niveles séricos de cortisol. Materiales y métodos: se realizó un estudio exploratorio correlacional. La información se obtuvo de 116 profesores a quienes se aplicó un instrumento estandarizado, se realizó un examen médico y se tomó una muestra de sangre para medir los niveles de cortisol. Resultados: El 51.8\% de los docentes analizados presentó riesgo psicosocial alto o muy alto asociado con la actividad desarrollada, un 31\% no mostró riesgo y un $17.2 \%$ presentó riesgo bajo. No se encontró relación entre los niveles de riesgo intralaboral y los niveles séricos de cortisol de los participantes. Conclusiones: este estudio no encontró relación entre ningún parámetro de riesgo evaluado y los niveles séricos de cortisol en la población estudiada.

(Fajardo-Zapata A, Montejo-Angel F, Molano-Vargas G, HernándezNiño J, Quintero-Guzmán A, 2013. Correlación entre los Factores de Riesgo Intralaboral y los Niveles Séricos de Cortisol en Docentes Universitarios. Cienc Trab. Ene-Abr; 15 [46]: 1-6).

Palabras claves: ESTRÉS LABORAL, DOCENTE, CORTISOL, RIESGO LABORAL.

\section{INTRODUCCIÓN}

Actualmente se sabe que la forma de la organización empresarial y las condiciones internas de trabajo tienen una influencia sobre el estado de salud de quienes laboran al interior de las organiza-

Correspondencia / Correspondence

Dr. Álvaro Luis Fajardo-Zapata

Universidad Nacional de Colombia

Fundación Universitaria del Área Andina

Carrera $14 \mathrm{~N}^{\circ}$ 69-35, Bogotá, Colombia

Tel.: (57-01) 2126320

e-mail: afajardo@areandina.edu.co

Recibido: 15 Enero 2013 / Aceptado: 02 de Febrero 2013

\section{ABSTRACT}

Introduction: the professors face up different generating job stress factors. Objective: to explore the association between the factors of psicosocial intralaboral risk and the blood levels of cortisol. Materials and methods: A correlacional exploratory study had carried out. The information was obtained of 116 professors, a standardized instrument was applied to them, a medical examination was carried out and a sample of blood was tooked to measure the levels of cortisol. Results: 51.8\% of the participants qualified on high or very high risk, in these two cases with $25.9 \%$ respectively; the $31 \%$ qualified without risk (17\%) or low risk (14.8\%) and 16.3\% corresponded to medium risk. One did not find relation between the levels of risk intralaboral and the levels séricos of cortisol of the participants. Conclusions: this study did not find relation between any evaluated risk parameter and the blood levels of cortisol in the study population.

Key words: BURNOUT, PROFESSOR, FACULTY, HYDROCORTISONE, OCCUPATIONAL RISKS. ciones; influencia que puede verse reflejada en el estado de salud mental y puede afectar tanto el desarrollo de la actividad laboral como la calidad de vida de los individuos. ${ }^{1}$

Dentro de este marco de análisis e intervención en los problemas de salud mental laboral, los factores de riesgo psicosocial intralaboral ocupan un lugar destacado. Desde hace varias décadas la psicología norteamericana ha realizado múltiples estudios en esta área buscando contribuir a dilucidar esta problemática del ámbito laboral. Las empresas también han contribuido a resolver esta problemática para mejorar la calidad de vida de los individuos al interior de las organizaciones a través de programas para prevenir el deterioro de la salud mental, y así mejorar la percepción que tienen los individuos acerca de sí mismos, de su rol y su aporte en el desarrollo y crecimiento de las organizaciones. ${ }^{1}$

Si bien la regulación colombiana sobre el tema de los riesgos psicosociales tiene un marco claro de acción desde el año 2008 en la resolución 2646 de 2008, es a partir de 2010 cuando se dispone 
de herramientas válidas y confiables que permiten conocer el comportamiento de este fenómeno., ${ }^{2,3}$

La población docente de las instituciones educativas se enfrenta a diferentes situaciones asociadas con los factores de riesgo psicosocial; sin embargo, aún en el país no se dispone de estudios que pongan a prueba el modelo de riesgo psicosocial y sustenten la resolución 2646, de igual manera no existen publicaciones que evidencien la aplicación y la pertinencia de la batería de pruebas de evaluación de factores de riesgo psicosocial y, específicamente, el riesgo intralaboral en docentes universitarios.

Por otra parte, la literatura ha evidenciado una relación directa entre los niveles séricos de cortisol y la presencia de estrés crónico en diferentes poblaciones; sin embargo, no se ha verificado la relación entre dicho marcador biológico y los factores de riesgo psicosocial intralaboral en docentes universitarios colombianos.

\section{LOS RIESGOS PSICOSOCIALES}

La calidad de vida abarca el bienestar físico, social, psicológico y espiritual. El bienestar físico se determina por la actividad funcional, la fuerza o la fatiga, el sueño y el reposo, el dolor y otros síntomas. El bienestar social, tiene que ver con las funciones y las relaciones, el afecto y la intimidad, la apariencia, el entretenimiento, el aislamiento, el trabajo, la situación económica y el sufrimiento familiar. El bienestar psicológico se relaciona con el temor, la ansiedad, la depresión, la cognición y la angustia que genera la enfermedad y el tratamiento. Por último, el bienestar espiritual abarca el significado de la enfermedad, la esperanza, la trascendencia, la incertidumbre, la religiosidad y la fortaleza interior. ${ }^{4}$

El trabajo es una condición inherente al ser humano, que se hace necesaria y lo acompaña durante gran parte de su vida; a través de él, las personas pueden llegar a satisfacer necesidades de todo tipo, desde las que tienen que ver con su supervivencia hasta las que le pueden llevar a sentirse autorrealizado. Dada su naturaleza le debiera ser gratificante, debería representarle la oportunidad de trascender más allá de su propia existencia. Sin embargo, el trabajo puede ser vivido también como una condición poco satisfactoria que puede traer consigo diversas repercusiones para quien así lo perciba. La razón de esta situación puede deberse a un sinnúmero de factores que van desde el trabajo en sí hasta las condiciones que predominan tanto a nivel de la organización como del entorno. Actualmente se viene generando la aparición de nuevos riesgos para la salud de los trabajadores, que se suman a los ya tradicionales; estos son los riesgos psicosociales, que tienen su origen en la organización del trabajo. ${ }^{5}$

Un informe publicado por la Agencia Europea para la Seguridad y la Salud en el Trabajo sobre riesgos psicosociales revela que los cambios técnicos $\mathrm{u}$ organizativos en el mundo laboral, junto con los cambios socioeconómicos, demográficos y políticos, incluido el fenómeno de la globalización, han originado riesgos psicosociales que están teniendo consecuencias sobre la salud de la población debido a que pueden provocar un mayor estrés laboral y repercutir en el bienestar de los trabajadores. Estos riesgos han sido agrupados en cinco áreas: a) nuevas formas de contratación laboral, que se caracterizan por la aparición de contratos de trabajo más precarios y la subcontratación e inseguridad en el puesto de trabajo; b) envejecimiento de la población activa, que la hace más vulnerable a la carga mental y emocional; c) intensificación del trabajo, caracterizado por la necesidad de manejar cantidades de información cada vez mayores bajo una mayor presión en el ámbito laboral; d) fuertes exigencias emocionales en el trabajo, junto con un incremento del acoso psicológico y de la violencia, en especial en los sectores de la asistencia sanitaria y de los servicios en general; y. e) desequilibrio y conflicto entre la vida laboral y personal, debido a la falta de ajuste entre las condiciones de trabajo y contratación y la vida privada de las personas. ${ }^{6}$

Desde finales del siglo pasado los factores psicosociales son uno de los temas que mayor atención y preocupación han causado entre los involucrados en el tema de la salud, seguridad e higiene ocupacional. Dichos factores representan un riesgo que tiene relación con las exigencias laborales y cuyas consecuencias no son tan evidentes, pero afectan la salud del trabajador. ${ }^{5}$

Los riesgos psicosociales se han definido por la Organización Internacional del Trabajo OIT como "las interacciones entre el contenido, la organización y la gestión del trabajo y las condiciones ambientales, por un lado, y las funciones y necesidades de los trabajadores, por otro". Estas interacciones podrían ejercer una influencia nociva en la salud de los trabajadores a través de sus percepciones y experiencia. Es una idea generalizada en la sociedad contemporánea que una de las consecuencias sobre la salud del individuo generada por el trabajo es el estrés, el cual le puede llegar a producir graves problemas. ${ }^{5}$

Es función de la salud ocupacional identificar, valorar y controlar los factores de riesgo psicosocial, y la planificación de medidas preventivas correspondientes que conduzca a una gestión eficaz de las personas de las organizaciones, que se traducirá en eficacia para la organización, mejor rendimiento, menor ausentismo laboral y, por supuesto, mayor satisfacción.

El manejo de los riesgos laborales de origen psicosocial es todavía incipiente en cuanto a la prevención de riesgos y enfermedades ocupacionales; sin embargo, cada día se constata más la importancia que tienen estos sobre la salud de los trabajadores y de las organizaciones.

Según la encuesta nacional de salud de España, los trabajadores que se perciben expuestos a factores de riesgo psicosocial (por ejemplo, sobrecarga de trabajo, exceso de carga mental, realización de tareas repetitivas y de muy corta duración) presentan porcentajes de respuesta significativamente mayores en sintomatología psicosomática (problemas de sueño, cansancio, dolores de cabeza, mareos, etc.) que los no expuestos. ${ }^{7-9}$

Según esta misma encuesta, el 22,5\% de los trabajadores considera que el trabajo está afectando su salud. Las manifestaciones que con más frecuencia atribuyen los encuestados al trabajo son: el dolor de espalda (57,6\%), el dolor de cuello $(28,1 \%)$, y el estrés $(27,9 \%)$. En relación con la presencia de otros sintomas, el cansancio, las alteraciones del sueño y las cefaleas inciden de forma importante sobre la población trabajadora, afectando al $12,3 \%, 12 \%$ y 10,4\%, respectivamente..$^{7-9}$

En Colombia, de acuerdo con la información reportada en la primera encuesta nacional sobre condiciones de salud y de trabajo, realizada en 2007, la prevalencia de exposición a factores de riesgo psicosocial es alta. Estos agentes se constituyen en los percibidos con mayor frecuencia por los trabajadores, superando incluso a los ergonómicos. La atención al público y el trabajo monótono y repetitivo ocupan los dos primeros lugares en más del 50\% de los encuestados. La encuesta en mención se realizó 
en empresas de diferentes actividades económicas y regiones del país, afiliadas al sistema general de riesgos profesionales. ${ }^{10}$

Con respecto al estrés ocupacional, entre el 20\% y el 33\% de los trabajadores manifestó sentir altos niveles de estrés. Asimismo se evidenció que los agentes ergonómicos y psicosociales fueron los más relacionados con la ocurrencia de los accidentes de trabajo. ${ }^{10}$

Los factores psicosociales comprenden los aspectos intralaborales, extralaborales o externos a la organización y las condiciones individuales o características intrínsecas al trabajador, los cuales en una interrelación dinámica, mediante percepciones y experiencias, influyen en la salud y el desempeño de las personas. $^{3}$

Las condiciones intralaborales son entendidas como aquellas características de trabajo y de su organización que influyen en la salud y bienestar del individuo tales como: demandas de trabajo, control sobre el trabajo, liderazgo y relaciones sociales en el trabajo y recompensa. ${ }^{3}$

Las condiciones extralaborales comprenden los aspectos del entorno familiar, social y económico del trabajador. A su vez, abarcan las condiciones del lugar de vivienda, que pueden influir en la salud y bienestar del individuo. En este aspecto se consideran: tiempo fuera del trabajo, relaciones familiares, comunicación y relaciones interpersonales, situación económica del grupo familiar, características de vivienda y de su entorno, influencia del entorno extralaboral sobre el trabajo y desplazamiento y vivienda, trabajo-vivienda. ${ }^{3}$

Las condiciones individuales aluden a una serie de características propias de cada trabajador o características socio-demográficas como el sexo, la edad, el estado civil, el nivel educativo, la ocupación (profesión u oficio), la ciudad o lugar de residencia, la escala socio-económica (estrato socio-económico), el tipo de vivienda y el número de dependientes. Estas características socio-demográficas pueden modular la percepción y el efecto de los factores de riesgo intralaborales y extralaborales. ${ }^{3}$

$\mathrm{Al}$ igual que las características socio-demográficas, existen unos aspectos ocupacionales de los trabajadores que también pueden modular los factores psicosociales intra- y extralaborales, tales como la antigüedad en la empresa, el cargo, el tipo de contratación y la modalidad de pago, entre otras. ${ }^{3}$

\section{EL ESTRÉS Y LOS NIVELES DE CORTISOL}

La activación del eje hipotálamo-hipófisis-suprarrenal es una de las respuestas más características del estrés generado por los riesgos psicosociales a los cuales está sometido el individuo. Los niveles de cortisol (sanguíneo, urinario o salival) son un buen marcador de la respuesta al estrés, aunque la reactividad del cortisol ante estresores situacionales no está exenta de problemas en su interpretación. ${ }^{11}$

Se ha descrito hipercortisolemia en situaciones de alto estrés en médicos de urgencias durante las horas de trabajo, aunque otras investigaciones muestran que los niveles de cortisol no se afectan como reflejo bioquímico del estrés en estos mismos profesionales. Estudios que han evaluado el estrés y los niveles de cortisol no han encontrado diferencias en los niveles séricos de la hormona entre la jornada laboral y la no laboral o en los periodos de tiempo donde no se trabaja. Estos resultados negativos van contra la hipótesis que postula que el estrés laboral tiene efectos sobre el cortisol; éste debe estar más elevado en días laborales o en los momentos en los que se está trabajando respecto a jornadas no laborales o tiempos de descanso. ${ }^{12-14}$

Recientemente, y a la vista de los resultados contradictorios mencionados, se ha sugerido que es posible que el cortisol medido durante el trabajo no sea un buen marcador de estrés crónico. La respuesta matutina de cortisol se asocia principalmente a estresores crónicos, mientras que las variaciones a lo largo del día se asocian con estado de ánimo negativo y estrés percibido. De esta forma, el interés se está centrando en la respuesta matutina del cortisol salival como indicador de estrés crónico, estrés laboral y depresión, habiéndose sugerido que un decremento en dicha respuesta matutina puede ser un marcador de una disfunción adrenocortical, lo que podría actuar como un factor de vulnerabilidad a ciertos síndromes físicos $\mathrm{y}$ mentales. ${ }^{15,16}$

El cortisol, y más en concreto la respuesta matutina del cortisol salival al estrés laboral también parece estar mediada por el género, ya que dicha respuesta es mayor en mujeres en comparación con hombres. Estas diferencias han sido confirmadas, tras comparar días laborales y no laborales, describiéndose una mayor respuesta matutina de cortisol durante los días laborales en mujeres que en hombres. Estas diferencias entre géneros podrían deberse a la mayor carga doméstica que tienen las mujeres, incluyendo el cuidado de los niños. ${ }^{11,15}$

En México, un estudio en profesores de secundaria mostró diferencias entre mujeres y hombres en los niveles de cortisol en saliva. El análisis de los factores psicosociales resaltó la asociación en mujeres entre el hostigamiento psicológico y los niveles de cortisol y en los hombres su asociación con el estrés. En este estudio el incremento del cortisol en saliva aparentemente se asoció, más que con el carácter estresante de la actividad docente, con condiciones que agreden al individuo o que son el efecto de la exposición crónica a los riesgos psicosociales. ${ }^{17}$

Moya y otros compararon la satisfacción laboral y la respuesta matutina de cortisol en profesores con altas y bajas puntuaciones en Burnout. El estudio no observó correlaciones significativas entre los niveles de cortisol y la satisfacción laboral. ${ }^{18}$

Así, la hormona que ha recibido más atención relacionada con los riesgos psicosociales es el cortisol, siendo generalmente medido en saliva. Se han descrito elevaciones vespertinas de esta hormona en ejecutivos que tenían sintomas de padecer Burnout. Se han descrito niveles mayores de cortisol matutino en sujetos que puntuaban alto en Burnout o tenían fatiga crónica. En las investigaciones donde se han estudiado los niveles de cortisol en sangre no se han encontrado las relaciones descritas. En muestras de orina, se ha descrito una hipocortisolemia en mujeres sudafricanas con el sindrome de Burnout. ${ }^{19-24}$

\section{LOS PROFESORES Y LOS RIESGOS PSICOSOCIALES}

Trabajar como docente tiene aspectos que suponen una exigencia emocional con potencial efecto sobre el estado de salud; también los docentes están expuestos a los riesgos generales. En este sentido, el informe de Fasecolda (federación aseguradores colombianos) de 2010 mostró que el 1\% de la totalidad de enfermedades profesionales calificadas en Colombia se registró en trabajadores de la educación universitaria. ${ }^{25}$

En el ámbito de la salud ocupacional aún existen aspectos que al 
parecer no han sido suficientemente explorados o dilucidados como ocurre con los factores psicosociales. Estos se han pasado por alto a pesar de que tienen una importante relación con la salud laboral, ya que los mecanismos con que el trabajador afronta y confronta las exigencias propias del medioambiente ocupacional en sus distintos niveles (organizacional, grupal e individual) ocasionan estrés y éste se refleja en su salud mental, su desempeño y satisfacción laboral. Entre ellos se encuentran los relacionados con el contenido de trabajo, el grado de responsabilidad, el conflicto, la ambigüedad del rol, los contactos sociales, el clima de la organización, el contacto con alumnos, la carga de trabajo, los horarios irregulares, la violencia en los espacios laborales, el ambiente físico en el que se realiza el trabajo y el no tener oportunidad de exponer la quejas e inseguridad en el empleo; estos elementos pueden incidir sobre la salud y la satisfacción de los docentes. Por otra parte, el docente puede estar sometido a determinadas exigencias para cumplir el plan y programas con eficiencia y eficacia, además de escasez de herramientas y recursos necesarios, lo que hace que esta profesión sea considerada como una de las más estresantes. ${ }^{26}$ Los riesgos psicosociales existentes en el ámbito universitario son el resultado de las interacciones que se producen entre sus características (pública, privada, etc.) y las demandas y condiciones de trabajo (formas de organizar el trabajo, tipo de tareas, relaciones laborales, etc.) con las necesidades, habilidades y expectativas que tiene el profesor universitario (estabilidad laboral, recursos, promoción, etc.). La combinación de estas características y su grado de exposición pueden afectar la salud de los docentes. ${ }^{27}$

La percepción de una inadecuada retribución, excesiva burocratización, tiempo dedicado a la preparación de las clases, tareas relativas a la docencia que realiza en casa, horario, problemas con los estudiantes, etc. se citan entre los desencadenantes de estas situaciones. ${ }^{28}$

Por otro lado, actualmente la universidad se encuentra ante nuevos retos que exigen incrementar la calidad de la docencia y la excelencia en la investigación. Todo ello puede manifestarse en un incremento de las demandas y exigencias hacia el profesorado, a las que probablemente deba hacer frente sin tener los recursos suficientes, lo que configura una situación idónea para la aparición del estrés laboral. ${ }^{29}$

Cuando el profesorado universitario percibe su situación como estresante con base a las caracteristicas organizativas que delimitan sus tareas (tanto en el sistema relacionado con el entorno social, físico-tecnológico, social-interpersonal o en el personal), y en función de sus capacidades de afrontamiento y/o de las condiciones de trabajo, el resultado puede derivar en la afectación de su rendimiento laboral y su salud. ${ }^{30,31}$

La evaluación de riesgos que debe realizarse como primer paso de la actividad preventiva debería basarse en estos marcos generales que han mostrado relación con el estado de salud, adaptarlos a la realidad de los docentes y añadir algunas dimensiones específicas. Sería deseable efectuar una detección precoz de los estados iniciales de las diversas situaciones de estrés que se pueden generar en la universidad, y en las que estarán implicadas variables sociodemográficas, organizativas y de personalidad, y que puedan producir desencadenantes negativos. Con ello también se evitaría el gasto económico y de personal que sufren las administraciones educativas por causa del ausentismo. ${ }^{31}$

Con relación al profesorado universitario los riesgos psicosociales que le pueden estar afectando hacen referencia a: ${ }^{31}$

- Las condiciones presentes en su situación laboral y contexto universitario (retribución, estabilidad laboral, políticas educativas, etc.).

- A la naturaleza de su trabajo y a sus requerimientos (carga mental, gestión emocional, etc.).

- Al modo habitual de desempeño y/o realización de la tarea (relaciones, equipos, presión, etc.).

- En muchos casos, las condiciones de contratación y la exposición a la necesidad de pluriemplearse, se constituyen en factor de exposición al riesgo psicosocial. ${ }^{32}$

\section{MATERIALES Y MÉTODOS}

Estudio exploratorio correlacional. Consistió en evaluar el riesgo psicosocial de un grupo de docentes universitarios mediante la aplicación de la batería para la evaluación de factores de riesgo psicosocial elaborada por el Ministerio de Salud de Colombia, que fue diligenciada por cada uno de los participantes del estudio; además, a los docentes se les prácticó un examen médico, se les tomó una muestra de sangre venosa en el antebrazo para determinar los niveles de cortisol (la muestra de sangre fue tomada a las 7 a. m.). El análisis de sangre para la determinación de los niveles de cortisol en suero fue realizado por la técnica de ELISA. Los docentes seleccionados fueron escogidos dentro del grupo de los presenciales. Para determinar si había relación entre el nivel de estrés de los docentes y los niveles de cortisol se realizó la correlación de rango de Spearman, para el cálculo de la respectiva correlación se utilizó el programa estadístico SPSS. Como universo se tomó a todos los docentes que tuvieran contrato laboral y que llevaran más de un año en la institución. El tamaño de la muestra fue de 116 docentes. Los participantes fueron seleccionadas mediante muestreo aleatorio simple. Se consideró un 10\% de pérdida por no participación voluntaria. Se tomó como error el cinco por ciento (5\%), una prevalencia estimada del 15\% (que corresponde a estudios previos en el mundo) y la población de docentes que fue de 300. El manejo de la información de las encuestas fue anónimo y confidencial. La investigación se llevó a cabo respetando en todo momento las directrices de la declaración de Helsinki y en atención a la dignidad humana y a los derechos de las personas, asimismo con la firma del debido consentimiento informado de los participantes.

\section{RESULTADOS}

La muestra estudiada correspondió a 116 profesores de ambos sexos distribuidos en 25 dependencias de la Fundación Universitaria del Área Andina. El programa de Enfermería fue el que más aportó unidades de estudio con 18 sujetos que correspondieron al 15.5\% del total. El grupo etario con mayor participación fue el comprendido entre 31 y 35 años con el 18.5\% y el de menor fue el de más de 55 años con el 4.4\%. El 62\% de los participantes correspondió al sexo femenino y $37.8 \%$ al sexo masculino. El $77.8 \%$ de los participantes tenía contrato a término fijo, el 5.9\% con contrato indefinido y $2.2 \%$ era de cátedra.

El análisis de las condiciones intralaborales de los profesores de este estudio mostró que el 51.8\% (60/116) de los docentes analizados tenía alto riesgo psicosocial asociado con la actividad desarrollada, un 31\% (40/116) no mostró riesgo y un $17.2 \%$ (16/116) presentó riesgo bajo. Dentro de ese riesgo encontrado el 
componente que más participó para que se presentara esta situación fue el de liderazgo, con el 48,1\% y las demandas en el trabajo con el 45\%. Asimismo, la dimensión retroalimentación del desempeño mostró un nivel de riesgo muy alto, con el 52,6\% de expuestos. En el control sobre el trabajo, en la dimensión control y autonomía sobre el trabajo, mostró que los docentes tuvieron un riesgo alto con el 50,4\%. Las demás variables analizadas tuvieron puntuaciones muy bajas, lo que evidenció que no existe riesgo o el riesgo es muy bajo.

$\mathrm{Al}$ realizar la correlación entre las condiciones intralaborales encontradas en los profesores y los niveles de cortisol como marcador biológico medido en sangre, este estudio no encontró una relación lineal positiva entre ningún parámetro de riesgo evaluado y los niveles séricos de dicha hormona. (Coeficiente de correlación de Spearman (Rho) = -0,009); igualmente, tampoco evidenció correlación entre la edad de los docentes participantes y los niveles de cortisol sérico. (Coeficiente de correlación de Spearman (Rho) = 0,331).

Tampoco se encontró asociación en los niveles generales de riesgo psicosocial intralaboral y el tipo de contrato de los respectivos docentes que participaron en este estudio $\left(\mathrm{p}=0,103\right.$; $\left.\mathrm{Chi}^{2}=12,570\right)$.

\section{DISCUSIÓN}

La batería diseñada por el Ministerio de Salud, como herramienta metodológica para evaluar la presencia de riesgos psicosociales que se aplicó en este estudio a un grupo de docentes universitarios, permitió evaluar el estado general del riesgo a que están sometidos estos profesionales. No se encontraron estudios en Colombia donde se aplicara esta misma metodología, que permitiera realizar la comparación con los hallazgos de esta investigación.

Los resultados de este estudio no evidenciaron relación entre ninguno de los parámetros de riesgo evaluado y los niveles séricos del cortisol medido en sangre. Dichos resultados difieren de los encontrados en un grupo de profesores en México por Tovalin et al, quien encontró relación entre el incremento del cortisol en saliva y la actividad docente; sin embargo, Moya et al., también en un grupo de profesores con altas y bajas puntaciones en Burnout, en España no encontraron ninguna correlación entre los niveles de la hormona y la satisfacción laboral. ${ }^{18}$ La asociación entre el estrés generado por la actividad laboral y la elevación de los niveles de cortisol no es clara, ya que mientras dicha asociación ha sido descrita en algunos profesionales como los de la salud, existen otros estudios que, analizando estos mismos profesionales, no han encontrado tal relación. ${ }^{12-14}$

Igualmente vale la pena resaltar que en las investigaciones donde se han estudiado los niveles de cortisol medidos en sangre no se ha encontrado asociación con los niveles de estrés; dichas asociaciones han sido descritas cuando se ha estudiado en saliva o en orina. ${ }^{19-24}$

Para futuros estudios, se requiere tener un seguimiento longitudinal durante el período académico, dado que la muestra tomada en este estudio se obtuvo centrados únicamente en un momento "medio" del semestre académico, y no se pudo observar el comportamiento de la percepción del riesgo psicosocial ni la respuesta psicobiológica asociada durante el ciclo laboral universitario, para evaluar el comportamiento a lo largo de los diferentes momentos en que las demandas laborales cambian y se agudizan, en particular asociado a los momentos de evaluación de estudiantes y de cierre de periodo, donde se concentra la carga laboral de los docentes. También es recomendable que los niveles de cortisol sean medidos en saliva o en orina.

\section{AGRADECIMIENTOS}

Los investigadores agradecen a Laura Carolina Fajardo Álvarez por la revisión del texto, sus aportes y la traducción al inglés del resumen.

\section{REFERENCIAS}

1. Rubio C. Fuentes de estrés, sindrome de Burnout y actitudes disfuncionales en orientadores de Instituto de Enseñanza Secundaria. [Tesis Doctoral]. Badajoz: Universidad de Extremadura; 2003.

2. Colombia. Ministerio de la Protección Social. Bateria de instrumentos para la evaluación de factores de riesgo psicosocial; Manual general. Bogotá: Ministerio de la Protección Social; 2010.

3. Colombia. Ministerio de la Protección Social. Resolución número 2646 de 2008. Factores de riesgo psicosocial en el trabajo. Bogotá: Ministerio de la Protección Social; 2008.

4. Vinaccia S, Orozco L. Aspectos psicosociales asociados con la calidad de vida de personas con enfermedades crónicas. Diversitas. 2005;1(2):125-137.

5. Ovalle $M$, Cruz 0 , Aldrete $M$. Los riesgos psicosociales y el Burnout en académicos de la universidad de ciencias y artes de Chiapas. Segundo Foro de las Américas en Investigación sobre Factores Psicosociales, estrés y salud mental en el trabajo; 2008 22-25 oct; Guadalajara, México. Guadalajara: Universidad de Guadalajara; 2009.
6. European Agency for Safety and Health at Work. Expert forecast on emerging psychosocial risks related to occupational safety and health. Luxembourg: Office for Official Publications of the European Communities; 2007.

7. Vázquez $A$, Pérez $D$, Moreno $R$, Arroyo F. Factores psicosociales motivacionales y estado de salud. Med Segur Trab. 2010;56(218):12-21.

8. España. Ministerio del Trabajo e Inmigración. Encuesta de Calidad de Vida en el Trabajo [en línea]. Madrid: Ministerio de Trabajo e Inmigración; 2007[consultado feb 2013]. Disponible en: http://www.mtin.es/estadisticas/ ecvt/welcome.htm

9. Gil-Monte P. Algunas razones para considerar los riesgos psicosociales en el trabajo y sus consecuencias en la salud pública. Rev Esp Salud Pública. 2009; 83(2):169-173.

10. Colombia. Ministerio de la Protección Social. Encuesta Nacional de Salud; 2007 [en línea]. Bogotá: Ministerio de Protección Social; 2008. [consultado jun 2012]. Disponible en: http://www.minsalud.gov.co/salud/Documents/ Valle\%20del\%20Cauca.pdf 
11. Serrano MA. Adaptación psicobiológica al estrés social en una muestra de profesores: cambios hormonales, cardiovasculares y psicológicos [Tesis Doctoral]. Valencia: Universitat de València; 2006.

12. Moya-Albiol L, Serrano MA, González-Bono $E$, Rodríguez-Alarcón $G$, Salvador A. Respuesta psicofisiológica de estrés en una jornada laboral. Psicothema. 2005; 17(2):205-211.

13. Walsh JJ, Wilding JM, Eysenck MW, Valentine JD. Neuroticism, locus of control, type a behavior pattern and occupation stress. Work \& Stress. 1997; 11(2):148-159.

14. González J, Fernández $M$, Molina R, et al. Riesgo psicosocial en el trabajo, estrés autopercibido y cortisol en saliva en una muestra de urgenciólogos. Emergencias. 2012;24:101-106.

15. Kunz-Ebrecht SR, Kirschbaum C, Marmot M, Steptoe A. Differences in cortisol awakening response on work days and weekends in women and men from the Whitehall II cohort. Psychoneuroendocrinol. 2004;29:516-528.

16. Meinlschmidt G, Heim C. Decreased cortisol awakening response after early loss experience. Psychoneuroendocrinol. 2005;30:568-576.

17. Tovalin $H$, Carreón $R$, Marroquín $R$, et al. Niveles de Cortisol por sexo en profesores de secundaria de la ciudad de México. $1^{\circ}$ congreso internacional y $4^{\circ}$ foro de las Américas en investigación sobre factores psicosociales, estrés y salud mental en el trabajo. 2012 17-20 oct.; Bogotá, Colombia. Bogotá: Universidad de los Andes-Facultad de Ciencias Sociales; 2012.

18. Moya L, Serrano M, Salvador A. Job Satisfaction and Cortisol Awakening Response in Teachers Scoring high and low on Burnout. Span J Psychol. 2010;13 (2):629-636.

19. Grossi G, Perski A, Evergard B, Blomkvist V, y Orth-Gomér K. Physiological correlates of burnout among women. J Psychosom Med. 2003;55:306-316.

20. De Vente W, Olff M, Van Amsterdam JGC, Kamphuis JH, Emmelkamp PMG. Physiological differences between burnout patients and healthy controls: blood pressure, heart rate, and cortisol responses. Occup Environ Med. 2003;60(1):54-61.

21. Melamed S, Ugarten U, Shirom A, Kahana L, Lerman Y, Froom P. Chronic burnout, somatic arousal and elevated cortisol levels. J Psychosom Res. 1999;6: 591-598.
22. Pruessner JC, Hellhammer DH, Kirschbaum C. Burnout, perceived stress, and cortisol responses to awakening. Psychosom Med. 1999;61:197-204.

23. Roberts $A D$, Wessely $S$, Chalder $T$, Papadopoulos $A$, Cleare AJ. Salivary cortisol response to awakening in chronic fatigue syndrome. Brit J Psychiat. 2004;184: 136-141.

24. Moch SL, Panz VR, Joffe BI, Havlik I, Moch JD. Longitudinal changes in pituitary-adrenal hormones in South African women with burnout. Endocrine. 2003;21(3):267-272.

25. Colombia: Federación de Aseguradores Colombianos (Fasecolda). Estadísticas de riesgos profesionales: Enfermedades profesionales 2000-2010 [en línea]. Bogotá: Fasecolda; 2011 [consultado ago 2012]. Disponible en: http://www. fasecolda.com/fasecolda/BancoConocimiento/R/riesgos_profesionales_-estadisticas_del_ramofinal/riesgos_profesionales_-_estadisticas_del_ramofinal.asp

26. Galván MG, Aldrete MG, Preciado L, Medina S. Factores psicosociales y sindrome Burnout en docentes de nivel preescolar de una zona escolar de Guadalajara, México. Revista de Educación y Desarrollo. 2010;14(julsep):5-11.

27. Artazcoz L, Molinero E. Evaluación de los factores de riesgo psicosocial combinando metodologia cuantitativa y cualitativa. Arch Prev Riesgos Labor. 2004; 7(4):134-142.

28. Greenglas ER. Introducción. Rev Psicol Trab Organ. 2005;21(1-2):5-14.

29. León J, Maria, Avargues M. Evaluación del Estrés Laboral en la Universidad de Sevilla. Informes, Estudios, Trabajos y Dictámenes. Sevilla: Universidad de Sevilla; 2004.

30. Gil-Monte P. El sindrome de quemarse por el trabajo (Burnout); una enfermedad laboral en la sociedad del bienestar. Madrid: Pirámide; 2005.

31. Moriana E. Estrés y Burnout en profesores. Int J Clin Health Psychol. 2004; 4(3):597-621.

32. Montejo F. Estrés y áreas de la vida laboral en docentes de profesiones de salud. Vol. 1. Madrid: EAE; 2012.

33. Decreto 1832 de 1994 y Decreto 2566 de 2009, por el cual se adop-ta la Tabla de Enfermedades Profesionales. Presidencia de la República de Colombia (7 jul 2009). 